\title{
A hybrid layer-multiplexing and pipeline architecture for efficient FPGA-based
} multilayer neural network

\author{
Yiping Dong ${ }^{1 \mathrm{a})}$, Ce $\mathrm{Li}^{1}$, Zhen Lin ${ }^{1}$, and Takahiro Watanabe ${ }^{1}$ \\ ${ }^{1}$ Graduate School of Information, Production and System, Waseda University \\ 2-7 Hibikino, Wakamatsu-ku, Kitakyushu, Fukuoka 808-0135, Japan \\ a) dongyiping@fuji.waseda.jp
}

Received February 1, 2011; Revised June 13, 2011; Published October 1, 2011

\begin{abstract}
This paper presents a novel architecture for an FPGA-based implementation of multilayer Artificial Neural Network (ANN), which integrates both the layer-multiplexing and pipeline architecture. Given a kind of FPGA to be used, the proposed method aims at enhancing the efficiency of resource usage of the FPGA and improving the forward speed at the module level, so that a larger ANN can be implemented on traditional FPGAs and also a high performance is achieved. Usually FPGA board is not changed for every applications, thus, we need not mind about the usage of it if the application can be implemented within the resource limitation. We developed a new mapping method from ANN schematic to FPGA by using this hybrid architecture, and also developed an algorithm to automatically determine the architecture by optimizing the application specific neural network topology. The experimental results show that the proposed architecture can produce a very compact circuit for multilayer ANN to meet resource limitation of a given FPGA. Furthermore, higher performance is obtained as compared with conventional methods.
\end{abstract}

Key Words: artificial neural network (ANN), FPGA, hybrid architecture, high performance, pipeline

\section{Introduction}

Artificial neural networks (ANNs) are characterized as an adaptive, robust and parallel computing model, which has the capability to learn by using examples and to approximate any given functions [1]. It has been widely applied to the fields of signal processing, speech synthesis, pattern recognition, and so on [2-4]. Most of these applications require high-speed computation to achieve the performance requirements. The traditional methods are executed by the general processors based on Von-Neumann architecture. It cannot attain the speed requirement when the network size becomes large. The area and design cost are also high by using the general processor which makes the commercial application of neural network impossible [5]. So it is necessary to develop such a custom high performance and small sized hardware architecture that can exploit the inherent parallelism of neural network models.

As described in [6], general hardware platforms for implementing ANNs are classified into some 
classes like digital [7], analog, hybrid [8], FPGA based [9], or optical implementations. Compared with other platforms, FPGA is one of the most suitable platforms to implement ANN as it offers the parallel computation and re-configurability [10]. A lot of research groups rely their applications on an FPGA method [11-13]. Other classifications including transfer function characteristics are: on-chip/off-chip, analog/digital, threshold/look-up table (LUT)/computation [6]. The work in this paper belongs to the class of FPGA platform, off-chip learning, digital neuron, and transfer function realized by LUT method.

J. Misra [6] pointed out that FPGA-based ANNs still have low performance and difficult implementation of large ANNs. Architectural efforts to improve the performance of FPGA-based ANNs are reported in $[14,15]$, while the resource reduction is highly required. As we know, the FPGA resource spent on one neuron is so high that it's hard to implement a whole ANN on a single FPGA chip. There are several solutions to overcome the problem of limited FPGA resource, such as the optimization of co-design [16], stochastic model [17] and multiplexing [18,19]. All these approaches focus on reducing the resource required for the neural network at the expense of processing speed. This drawback makes the FPGA-based ANN architecture not used for a lot of applications of ANN which require high performance.

By the development of semiconductor technology, available resources in a current FPGA chip become much higher, so that the resource constraints are relaxed. Therefore it is necessary to take the processing speed into consideration when designing the architecture for the ANN implementation on FPGA.

The purpose of this paper is to design a novel method to improve processing speed of an FPGA-based ANN by enhancing the efficiency of resource usage of the same FPGA board. Our proposed hybrid implementation method could achieve higher performance for an FPGA-based ANN. The proposed architecture presents an advantage in two basic respects over the previous reported implementations. The first one is the hybrid of layer multiplexing (LM) and pipeline, which can optimize both the resource requirement and speed. The layer multiplexing guarantees the resource required by neural network under the constraint of an adopted FPGA chip, and the pipelining between the layers can improve the speed. The second point is the algorithm to determine the optimal hardware architecture according to the neural network parameters such as the topology, data structure and so on. When the proposed method just implements one layer at a time, it is the same as layer-multiplexing (LM) method. When all layers of ANN are implemented at a time, it is the same as a pipeline method. Furthermore, our method can solve the resource limitation of a given FPGA, so that the FPGA board need not be changed for different applications. By using the proposed architecture, the resource of the FPGA board is efficiently used, and the drawback of low processing speed of the LM is overcome. Thus, the FPGA-based ANN with this architecture could be used for more applications which require high processing speed. Our method takes both the performance and the resource usage into account unlike other works.

Furthermore, our target is to design a general method applied to different FPGA boards. The method can be used not just for small-scale FPGA boards but also large-scale FPGA boards. For example, XC2VP20 board is used as an example to introduce the proposed method which can implement at most 20 neurons at a time. For another example, XC7A350T board is used as a large-scale board which can implement about 300 neurons at a time. Unfortunately, the on-chip learning is difficult to be integrated in the proposed architecture according to speed afford. Therefore, the on-chip learning is left as a future subject in this paper.

The rest of this paper is organized as follows: Sect. 2 describes the circuit design of the single neuron. Sect. 3 presents the network design and the details of the proposed architecture. Sect. 4 describes experiments to validate the advantages of the proposed method. Finally, Sect. 5 concludes the paper. 


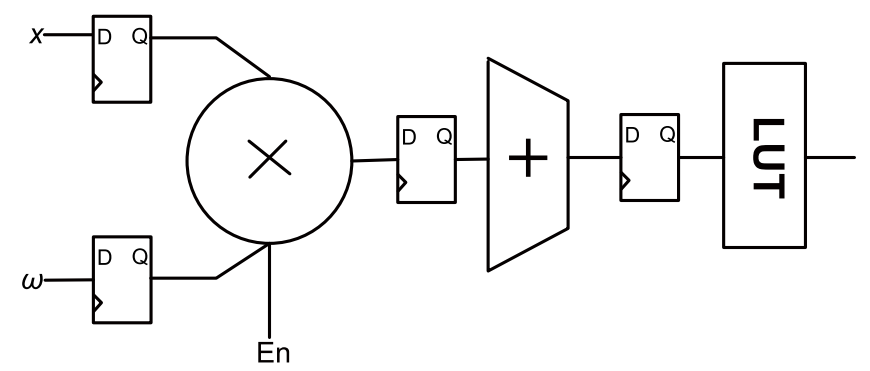

Fig. 1. Neuron circuit block diagram.

\section{Neuron design}

\subsection{Mathematical model}

A multilayer neural network is composed of one input layer, several hidden layers for computation and one output layer. Each layer consists of a set of processing elements called neurons and the main task of each neuron is processing the following function [20]:

$$
y=f(x)=f\left(\sum_{i=1}^{n} \omega_{i} x_{i}+b\right) .
$$

where $x_{i}$ stands for the $i^{t h}$ input, $\omega_{i}$ is the weight in the $i^{t h}$ connection, and $b$ is the bias. The function $f(x)$ is the nonlinear active function used in the neuron. Here we select the log-sigmoid as the active function due to its popularity [21] as described by the following:

$$
f(x)=\frac{1}{1+e^{-x}} .
$$

Because RAM is usually used to store the weights, we did not use the online learning in our proposed architecture, so that the hardware resource is saved.

\subsection{Neuron circuit design}

Some of hardware implementation of ANNs focus on the forward computing work and leave the learning work to computer as an off-chip learning, so does the FPGA-based ANNs. Hardware neuron design is classified into digital and analog methods. The FPGA-based ANN will use digital neuron design method. As shown in the above mathematical model and [22], the computational resources required by a single neuron are a multiplication block, an accumulation block and an active function block.

Figure 1 shows a block diagram of the neuron circuit, where En is an enable signal that controls the neuron's state, that is, whether the neuron is working or not. The selection of word length, that is, bit precision is important for the output resolution, where longer bits mean a higher resolution but also it takes a larger resource cost. In actually ANNs design, these parameters are set according to the application in order to achieve the efficient hardware implementation. In this paper, we consider the parameters as variables, which can be modified by users in the compilation step.

According to implementation of nonlinear activation function, there are lots of methods. In this work, the active function of digital neuron is realized by using LUT (look up table) [19] due to a large number of built-in RAMs supported in a modern FPGA chip. As the active function is highly nonlinear, a general procedure to obtain an LUT of the minimum size for a given resolution is as follows. As mentioned above, the bit length of output from the active function (Eq. (2)) is $N_{i}$.

1) The actual output of the active function is the value between $2^{-N_{i}}$ and $1-2^{-N_{i}}$ for the bit length $N_{i}$. Let $x_{1}$ and $x_{2}$ be the upper and the lower limits of the input range respectively, as Eq. (3). By solving Eq. (3), we get Eq. (4).

$$
\frac{1}{1+e^{-x_{1}}}=2^{-N_{i}}, \quad \frac{1}{1+e^{-x_{2}}}=1-2^{-N_{i}} .
$$




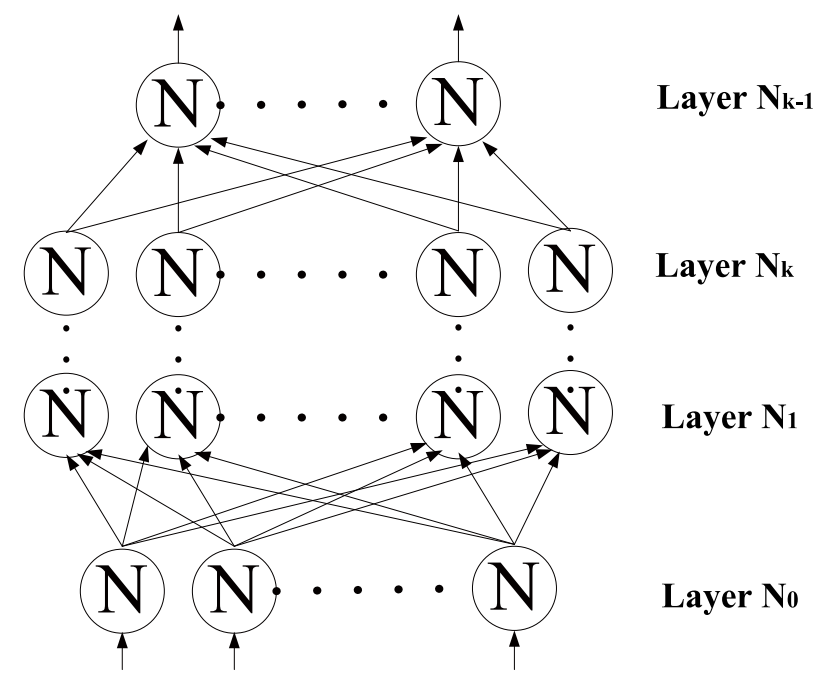

Fig. 2. Multilayer neural network.

$$
x_{1}=-\ln \left(2^{N_{i}}-1\right), \quad x_{2}=+\ln \left(2^{N_{i}}-1\right) .
$$

2) Consider the fact that the step change in the output $(\Delta y)$ is equal to $2^{-N_{i}}$, and the corresponding minimum change in input is at the point of maximum slope, $x=0$ in this case. So the minimum change value of input for the output change of $2^{-N_{i}}$ can be obtained from

$$
\Delta x=\ln \left(\frac{0.5+2^{-N_{i}}}{0.5-2^{-N_{i}}}\right) .
$$

3) The minimum number of LUT values is given by

$$
(L U T)_{\min }=\frac{x_{1}-x_{2}}{\Delta x} .
$$

\section{Network design}

Network connects all the neurons and all the layers together, so the data can be forwarded through the connections from the former layer to the latter layer. Figure 2 gives an example of a multilayer neural network.

Assume the symbol $x_{k}^{i j}$ and $y_{k}^{i}$ stand for the input and output of neurons, where the subscript of $k$ is the layer number and the superscript of $i$ is the neuron number in this layer and $j$ is the $j^{t h}$ input of this neuron. Suppose the ID for input layer is 0, then the forward process can be described as follows:

$$
\begin{gathered}
y_{1}^{i}=\sum_{j=1}^{N_{0}} w_{j i}^{01} \cdot x_{j}, \quad i \in\left[1, N_{1}\right], \quad j \in\left[1, N_{0}\right] . \\
x_{k}^{i j}=y_{k-1}^{j}, \quad y_{k}^{i}=\sum_{j=1}^{N_{k-1}} w_{j i}^{(k-1) k} \cdot x_{i j}, \quad k \in[2, N], \quad i \in\left[1, N_{k}\right], \quad j \in\left[1, N_{k-1}\right] .
\end{gathered}
$$

where $N_{k}$ is the total neuron number in layer $k$, and $w_{j i}^{(k-1) k}$ stands for the weight between the $j^{t h}$ neuron in layer $(k-1)$ and the $i^{t h}$ neuron in layer $k$, respectively.

There are two concept used in our proposed network architecture, including pipeline design and layer multiplexing design.

\subsection{Pipeline design}

The multilayer neural network has a characteristic that the neuron in the layer depends on the neurons in the previous layer and there is no communication among neurons in the same layer. Figures $3(\mathrm{a}-\mathrm{c})$ shows an example of in the conventional non-pipeline forward phase, where only one layer is working while the other layers are just waiting for the data coming from the previous layer. In order to save the 


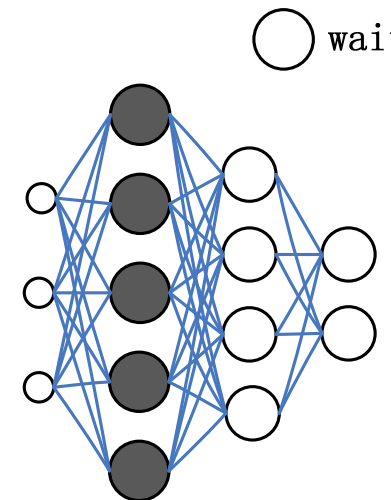

(a)

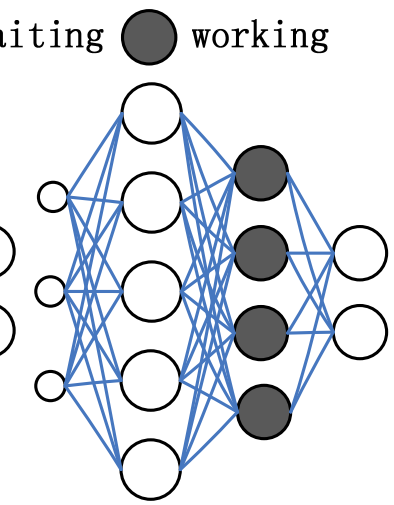

(b)

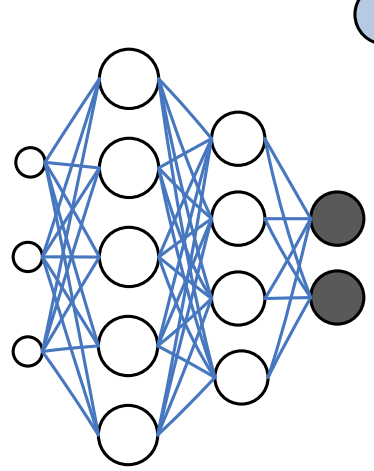

(c)

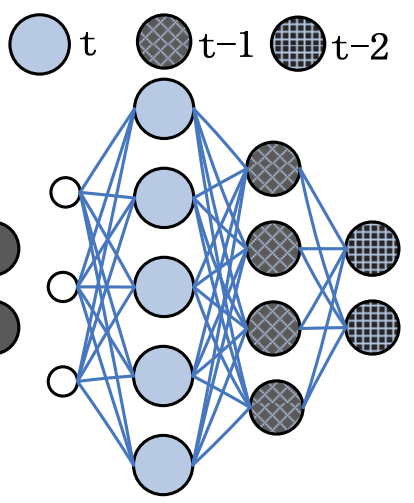

(d)

Fig. 3. Non-pipeline (a), (b), (c) vs pipeline (d).

cost, we integrate the pipeline manner in the layer architecture when forwarding. The fundamental pipeline algorithm is described as follows, where $\mathrm{t}$ is the time factor given by a clock cycle.

$$
\begin{gathered}
y_{1}^{i}(t)=\sum_{j=1}^{N_{0}} w_{j i}^{01} \cdot x_{j}(t), \quad i \in\left[1, N_{1}\right], \quad j \in\left[1, N_{0}\right] . \\
y_{2}^{i}(t)=\sum_{j=1}^{N_{1}} w_{j i}^{12} \cdot y_{1}^{j}(t-1)=\sum_{j=1}^{N_{1}} w_{j i}^{12} \cdot \sum_{k=1}^{N_{0}} w_{k j}^{01} \cdot x_{k}(t-1) . \\
y_{3}^{i}(t)=\sum_{j=1}^{N_{2}} w_{j i}^{23} \cdot y_{2}^{j}(t-1)=\sum_{j=1}^{N_{2}} w_{j i}^{23} \cdot \sum_{k=1}^{N_{1}} w_{k j}^{12} \sum_{m=1}^{N_{0}} w_{m k}^{01} \cdot x_{m}(t-2) .
\end{gathered}
$$

As shown in Fig. 3(d) and Eqs. (9)-(11), when the first layer is under computing for the input pattern at time $t$, the second layer is busy calculating the result which is transmitted from the first layer in the previous cycle $(t-1)$.

It doesn't need to wait for the end of some input pattern forwarding, but all the neurons in different layers are working simultaneously with different input pattern. By using the pipeline, the global forwarding speed would be much faster and there would not be much incidental cost or changes in the architecture, compared with a non-pipeline method. But just some modifications are needed, such as:

1) Enhance the clock rate for input memory module according to the pipeline depth.

2) Allocate a dependant memory for each neuron to store the weights.

3) Add a register to store the output of each neuron.

Unfortunately, it is hard to perform a whole neural network in pipeline manner by a popular FPGA chip due to limited FPGA resource. To solve this problem, we introduce the layer multiplexing into our pipeline method.

\subsection{Layer multiplexing with partly pipeline}

Layer multiplexing was first proposed by S. Himavathi in 2007 [19], which aimed at reducing the resource requirement in a multilayer neural network. Instead of realizing a complete network, only the single largest layer with each neuron having maximum number of input is implemented. The layer plays a role of different layers with the help of a control block. The control block ensures proper functioning by assigning the enable signal, appropriate inputs and weights for each neuron.

This method presents an advantage that it can substantially save the resource so that a larger network could be implemented in a single FPGA chip. But this is achieved at the expense of the forwarding speed, because there is only one layer being working and it has to reconfigure the network before performing neural computing in the next layer.

According to the experimental synthesis report of implementing ANNs by layer multiplexing, the utilization rate of slices in an FPGA is not always so high, that is, we still have enough slice resource 


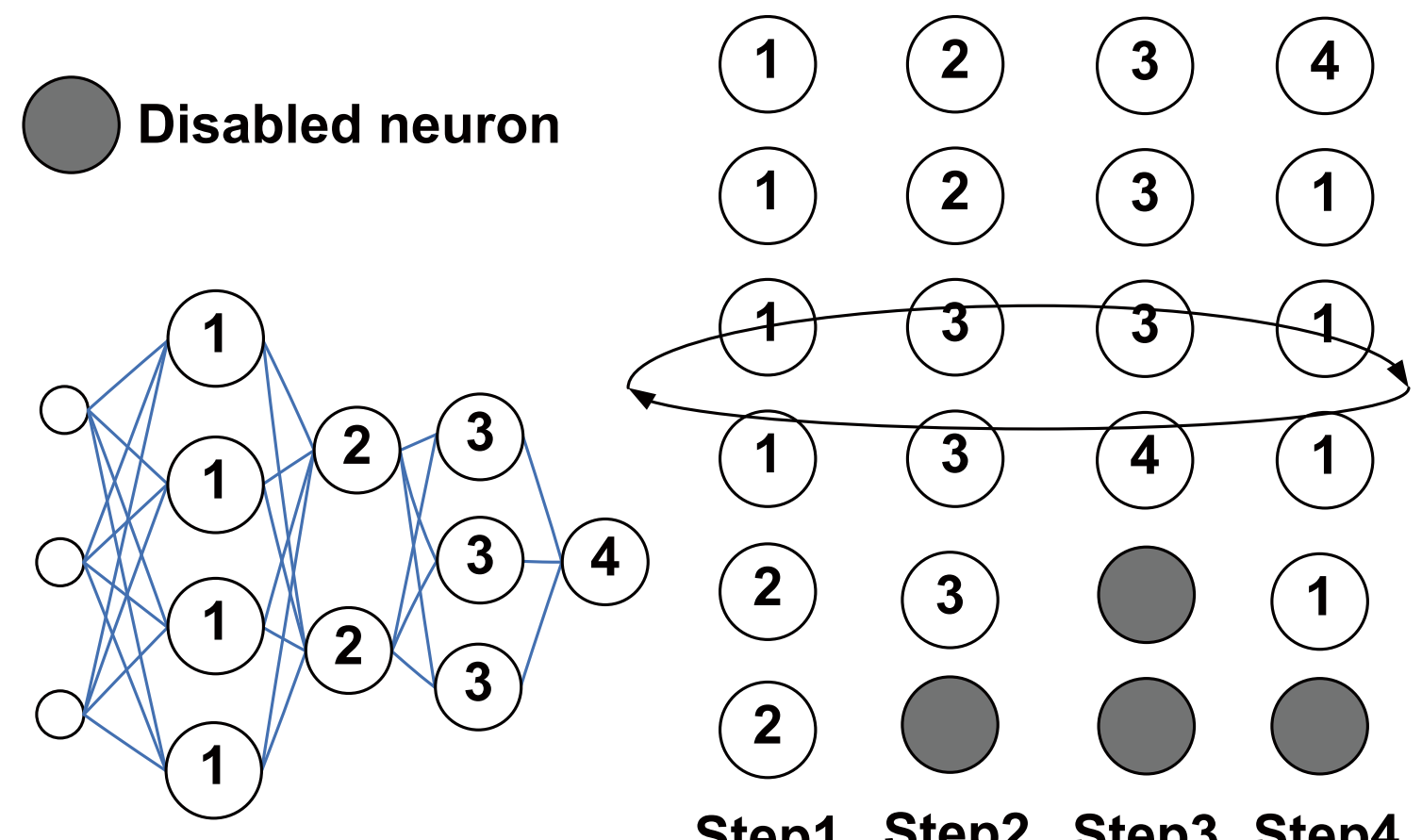

Fig. 4. Schematic of our mapping method.

to do some improvement to make the forwarding speed higher. The main idea in this paper is adding the partly pipeline manner to the layer multiplexing method.

In our proposed method, we first calculate the maximum number of neuron modules to fit an adopted FPGA chip. Then taking this value and the neural network topology into consideration, we can get an optimal solution by assigning the appropriate mapping method, pipeline depth and layer multiplexing. The optimal target is increasing the usage of chosen FPGA board to mapping more layers of ANN application to get high depth of pipeline, and then the high depth of pipeline will result high performance.

Figure 4 gives an example of our method. In this example, we suppose that the network topology is 3-4-2-3-1 and the maximum number of neuron modules in the FPGA is six. Numbers in the nodes in Fig. 4 mean the layer number. It performs the neural computing of adjacent two layers at a time with the pipeline manner between them. In Step1, the first two layers are under working, where the layer 1 is computing for the input pattern $a^{m+1}$ and the layer 2 is serving for input pattern $a^{m}$, respectively. In Step2, the $2^{\text {nd }}$ and $3^{\text {rd }}$ layers are configured by the layer multiplexing mechanism, where the $2^{\text {nd }}$ layer is busy with input pattern $a^{m+1}$ from the layer 1 in the previous step while the $3^{\text {rd }}$ layer is computing for input pattern am from layer 2 by layer multiplexing. The total number of neurons in the $2^{\text {nd }}$ and $3^{\text {rd }}$ layers is five, so there are disabled neuron modules to save the power. The succeeding steps are almost the same as the first two steps, including enable the proper number of neuron modules, get the corresponding input from previous step, perform neural computing for the incoming data and then send the result to the next step by layer multiplexing. When the computation in Step4 is completed, it would turn to the Step1, forming a loop. In this example, there are always two layers mapping the neuron modules in pipeline, which means the pipeline depth is two. By assigning different FPGA chip and neural network topology, the pipeline depth may be different.

\subsection{The control block design}

The operation of layer multiplexing and the partly pipeline are guaranteed by a control block, which is realized by the finite state machine (FSM). Figure 5 shows the whole circuit of neural network using our proposed method. The details of the block named $\mathrm{N}_{n}$ of the $n^{\text {th }}$ neuron is already presented in Fig. 1 in Sect. 2, so we will introduce the control block design here. The main task of the control block is assigning proper signals for the input and weight RAMs, the multiplexer and demultiplexer, 


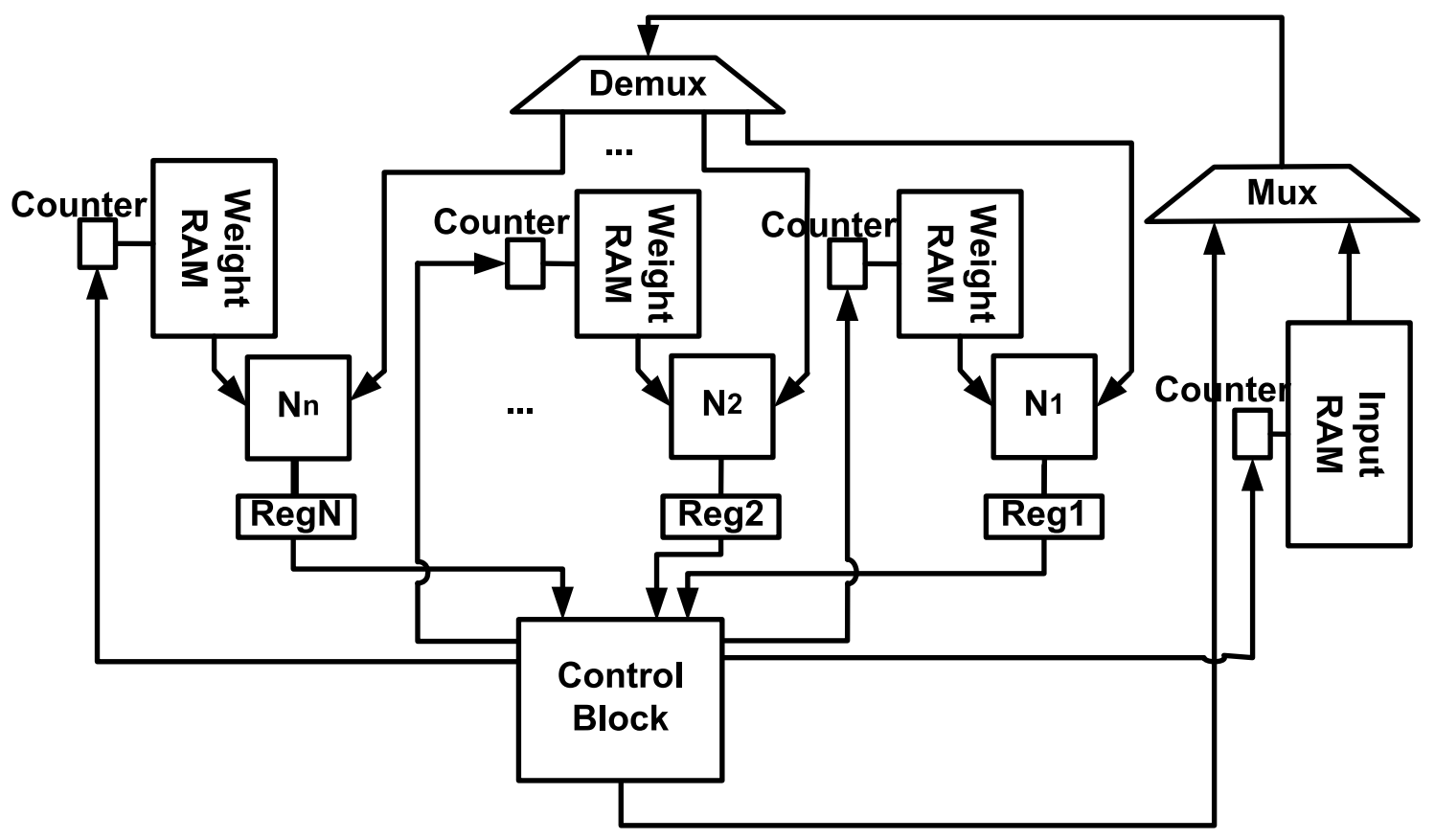

Fig. 5. The neural network circuit.

FSM (reset, clk, flag1 S, en1 N,con0 N, mux, demux) // input signal: reset, clk, flag1 S

// flag1 S: the finish signal of computation in Step s // en1 N: the enable signal for each neuron module // conO N: the address signals for each RAM // mux: the selection signal for the multiplexer // demux: the selection signal for the demultiplexer always (current_state or flag1 S)

case (current_state)

IDLE:

Step1:

$$
\text { Do }\{\ldots\}
$$

Do $\{$ set en $1 \sim N$, con0 N, mux, demux next_state $=$ Step 2$\}$

StepS:

Do $\{$ set en $1 \sim N$, con0 N, mux, demux next_state $=$ Step 1$\}$

default: next_step=IDLE endcase

\section{(a)}

Solve(topology, chiptype, datapath) // the topology information includes the number // of layers, the number of inputs, the number of // neurons in each layer // the data path contains the data representation // of input, weight that mentioned in section II.B.

1. begin

2. initial pipe_depth=layer_num

3. size=est_neuron_size( datapath)

4. max_num=cal_max_num(chiptype,size )

5. searching(pipe_depth, max_num, topology)

6. \{ stop_condition()

7. if (stop_condition())

8. searching(pipe_depth-1, max_num, topology)

9. else

10. return pipe_depth

11. endif\}

12. config_FSM(pipe_depth, topology, data_path)

13. end

\section{(b)}

Fig. 6. Control block (a) Pseudo code for the control block, (b) Procedure of topology generation.

Table I. Resource and performance of a neuron with different weight precisions.

\begin{tabular}{|c|c|c|c|c|c|c|c|}
\hline No. of bits & 10 & 11 & 12 & 13 & 14 & 15 & 16 \\
\hline Slices & 292 & 312 & 328 & 344 & 360 & 372 & 392 \\
\hline Max clk $(\mathrm{MHz})$ & 105.3 & 104.2 & 103.6 & 103.0 & 102.5 & 102.1 & 101.7 \\
\hline
\end{tabular}

and performing the logical data transmission. The pseudo code of the FSM is given in Fig. 6(a).

We also developed MATLAB program to determine the optimal architecture for a given neural network and a designated FPGA chip. Firstly, it estimates the number of slices used by a neuron module, given the data structure of the neuron. Secondly, the maximum amount of neuron modules 
Table II. Synthesis report for ANNs of 3-4-2-3-1 and 5-7-3-7-5 with VirtexII XC2VP20.

\begin{tabular}{|c|c|c|c|c|c|c|}
\hline \multirow{2}{*}{ Pipe Depth } & Network architecture & & \multicolumn{2}{|c|}{$3-4-2-3-1$} & \multicolumn{2}{|c|}{$5-7-3-7-5$} \\
\cline { 2 - 7 } & Features & Resources & Utilized & $\%$ & Utilized & $\%$ \\
\hline \multirow{3}{*}{2} & Slices & 9280 & 2764 & 29.8 & 5399 & 58.2 \\
\cline { 2 - 7 } & Flip Flops & 18560 & 2148 & 11.6 & 4195 & 22.6 \\
\cline { 2 - 7 } & LUTs with 4 input & 18560 & 5128 & 27.6 & 10011 & 53.9 \\
\hline \multirow{3}{*}{3} & Slices & 9280 & 4084 & 44.0 & 8375 & 90.2 \\
\cline { 2 - 7 } & Flip Flops & 18560 & 3400 & 18.3 & 6971 & 37.6 \\
\cline { 2 - 7 } & LUTs with 4 input & 18560 & 8036 & 43.3 & 16472 & 88.8 \\
\hline & Slices & 9280 & 4525 & 48.8 & $/ a$ & $/$ \\
\cline { 2 - 7 } & Flip Flops & 18560 & 3819 & 20.6 & $/$ & $/$ \\
\cline { 2 - 7 } & LUTs with 4 input & 18560 & 9008 & 48.5 & $/$ & $/$ \\
\hline
\end{tabular}

$a /:$ not enough slice for mapping 4 pipe depth.

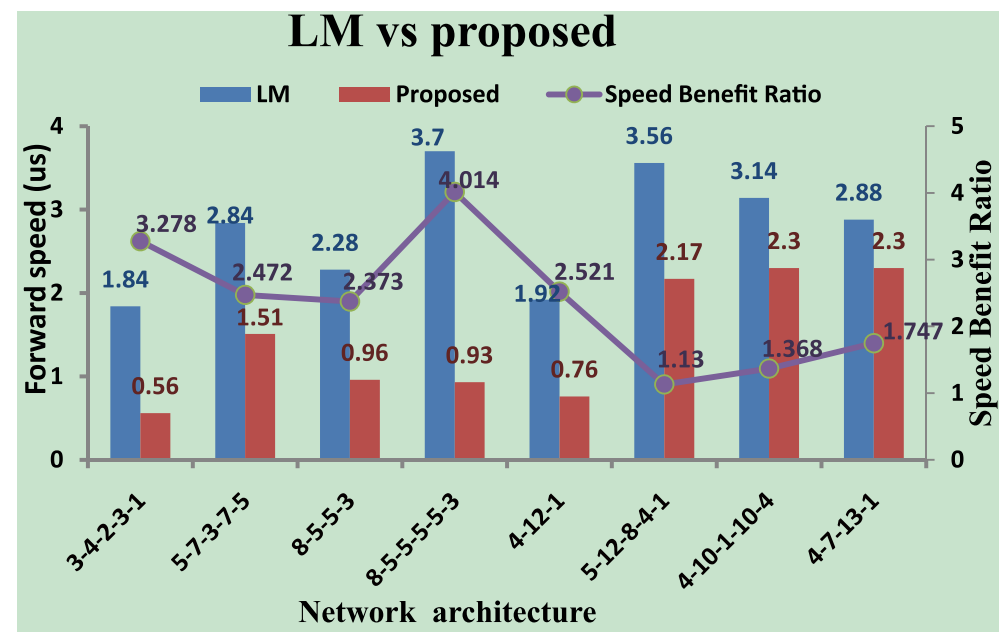

Fig. 7. Forward speed comparison of LM and proposed method.

allowed in the adopted FPGA chip can be figured out because we have integrated the information of several popular FPGA chip into the procedure. Then a searching function will be invoked to determine the optimal pipeline depth for the neural network. This procedure also provides the function that configures the parameters in the FSM. The pseudo code of the procedure is shown in Fig. 6(b).

\section{Experiments}

We use the Verilog HDL and Xilinx ISE 9.1i for design and synthesis. The simulation tool is performed by Modelsim XE II 7.3a. The FPGA chip we selected here is Xilinx VirtexII XC2VP20. The basic block of the VirtexII is the Logic Cell Block (LCB), which is composed of eight LUTs with four inputs, four slices, eight Flip-Flops, and so on [23].

Table I gives the synthesis report for a single neuron module by varying the data path in weights. From the table we can see that with the increase of data precision, the maximum frequency comes down a little. 16 bits are chosen in our simulation.

Table II shows the synthesis reports for two examples of neural network which mentioned in Sect. 3.2 and a pattern recognition application of ANN with topology of 5-7-3-7-5 [24]. The value of pipeline depth in the left column means the number of layers under execution at a time. Our proposed architecture is compact due to the simple module architecture and the effective control block, and also provides a flexible solution for a neural network to be implemented. Compared with other 
Table III. Comparsion between layer-mutiplexing and our method with various applications of ANN with device of XC2VP20.

\begin{tabular}{|c|c|c|c|}
\hline Network Architecture & $\begin{array}{c}\text { Implement method/ } \\
\text { Pipeline Depth }\end{array}$ & Slices & Utilization Rate (\%) \\
\hline \multirow{2}{*}{$3-4-2-3-1$} & LM & 1726 & 18.6 \\
\cline { 2 - 4 } & proposed / 4 & 4525 & 48.8 \\
\hline \multirow{2}{*}{$5-7-3-7-5[24]$} & LM & 2979 & 32.1 \\
\cline { 2 - 4 } & proposed / 3 & 8375 & 90.2 \\
\hline \multirow{2}{*}{$8-5-5-3[19]$} & LM & 2142 & 23.1 \\
\cline { 2 - 4 } & proposed / 3 & 5832 & 62.8 \\
\hline \multirow{2}{*}{$8-5-5-5-5-3[19]$} & LM & 2171 & 23.4 \\
\cline { 2 - 4 } & proposed / 4 & 8802 & 94.8 \\
\hline \multirow{2}{*}{$4-12-1[25]$} & LM & 5051 & 54.4 \\
\cline { 2 - 4 } & proposed / 2 & 5821 & 62.7 \\
\hline \multirow{2}{*}{$5-12-8-4-1[26]$} & LM & 5051 & 54.4 \\
\cline { 2 - 4 } & proposed / 2 & 8790 & 94.7 \\
\hline $4-7-13-1[28]$ & LM & 3870 & 41.7 \\
\cline { 2 - 4 } & proposed / 2 & 6262 & 94.7 \\
\hline \multirow{2}{*}{$4-1-10-4[27]$} & LM & 5464 & 58.9 \\
\hline & & 8775 & 94.5 \\
\hline
\end{tabular}

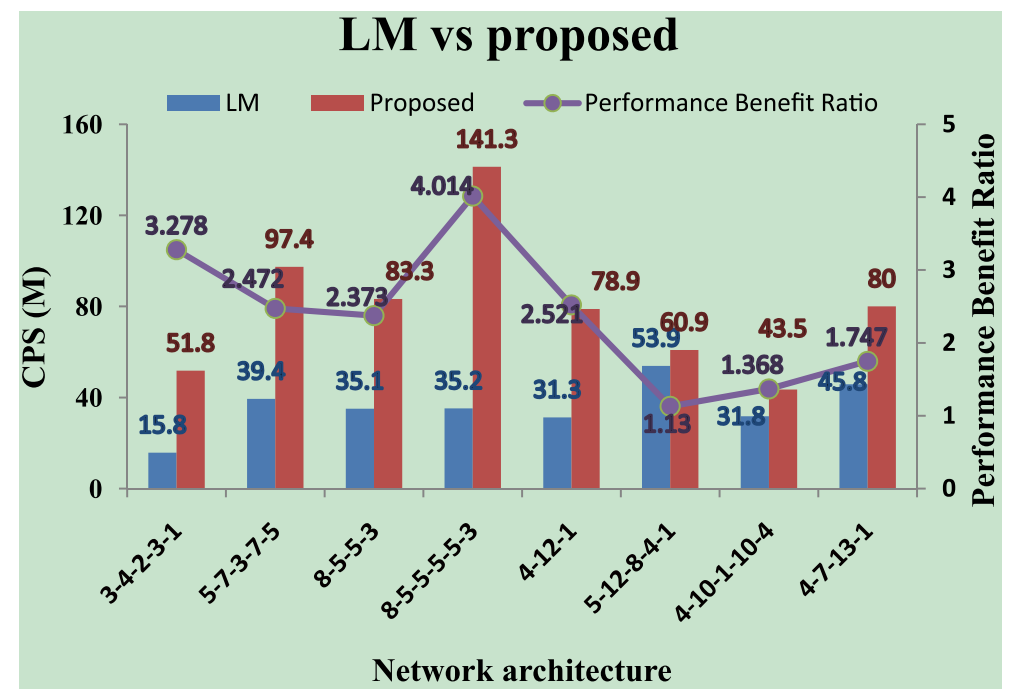

Fig. 8. Performance comparison of LM and proposed method.

blocks, the neurons blocks take much more resource. This FPGA board used in this simulation could implement at most 20 neurons. Implementing the ANN with topology of 5-7-3-7-5 with 4 pipeline depth needs 22 neurons, so that this ANN could not be implemented with 4 pipeline depth. That is, the pipeline depth is decided by the selected FPGA board and the network topology.

Table III shows a comparison between the traditional layer multiplexing method (LM) and our proposed method with different applications of ANNs [19,24-28]. We have integrated the partly pipeline manner into the layer multiplexing (the number in 'proposed/' means the pipeline depth). There are several layers mapping the neuron modules by pipeline manner while LM maps only one layer at a 
time. The forwarding speed is measured by the interval starting from one input pattern imported to get the result at the output layer. The Connection-Per-Second (CPS) is the most common performance measurement, which is defined as the rate of multiplication and accumulation operations [5]. The comparison results of forwarding speed and performance of CPS between LM method and the proposed method are shown in Figs. 7 and 8, respectively.

As a result, the forwarding speed of our proposed method is faster than that of LM with respect to the pipeline depth. Due to the pipeline manner, our method has much more neurons under working than LM, so it also shows an advantage in performance of CPS compared with LM method. The resource usage of the proposed method is increased compared with LM method, but the FPGA board is not changed. It means that our method could provide high performance for the given FPGA board.

\section{Conclusions and future work}

A general architecture for the implementation of a multilayer ANN was proposed. The circuit for each application can be easily generated by setting the parameter values to match the particular network size and running the synthesis. Similarly to a particular network, the best solution of the architecture design of pipeline and layer multiplexing is calculated by MATLAB procedure, by returning the optimal pipeline depth and the control signal value in each state of the control block (FSM). We exploited the capability of a given FPGA board by assigning the proper pipeline depth, so that a higher resource utilization rate, global forwarding speed and high performance were achieved.

Our proposed architecture makes an FPGA implementation easy for a given ANN at a short time by varying the data path. It also provides the feasibility to perform a larger neural network in a popular FPGA board at a relatively higher speed by using the partly pipeline method. So it is possible to develop a neural device for commercial or industrial application by our method. By using the proposed method, the drawback of low processing speed in the layer multiplexing method is overcome without increase of hardware. The FPGA-based ANN with the proposed method could be used for different applications which need high processing speed.

On-chip learning is another attractive property of a multi-layer neural network, but it is difficult to design for the proposed hybrid architecture and may decrease the speed. Thus, the on-chip learning problem is left as our future work.

\section{Acknowledgments}

We would like to thank anonymous reviewer and the Associate Editor for their helpful and constructive comments. This research was partly supported by Waseda University Global COE Program 'International Research and Education Center for Ambient SoC' sponsored by MEXT, Japan (GCOE), and also partly supported by Program for Fostering Regional Innovation Systems by MEXT, and Core Research for Evolutional Science and Technology (CREST), Japan Science and Technology.

\section{References}

[1] D. Graupe, Principles of Artificial Neural Networks, Advanced Series in Circuits and Systems, World Scientific, 2007.

[2] L. Jain, "Fusion of neural nets, fuzzy systems and genetic algorithms in industrial applications," IEEE Trans. Industrial Electronics, vol. 46, no. 6, pp. 1049-1136, 1999.

[3] D.S. Kim and J.S. Park, "Modeling network intrusion detection system using feature selection and parameters optimization," IEICE Trans. Information and Systems, vol. E91-D, no. 4, pp. 1050-1057, April 2008.

[4] J.F. Wang, J.C. Wang, A.N. Suen, C.H. Wu, and F.M. Li, "Vlsi architecture and implementation for speech recognizer based on discriminative bayesian neural network," IEICE Trans. Fundamentals, vol. E85-A, no. 8, pp. 1861-1869, August 2002.

[5] C.S. Lindsey, "Neural networks in hardware: Architectures, products and applications," lecture notes of Neural Networks, August 2002.

[6] J. Misra and I. Saha, "Artificial neural networks in hardware: a survey of two decades of progress," Neurocomputing, vol. 74, no. 1-3, pp. 239-255, 2010. 
[7] G. Dede and M.H. Sazli, "Speech recognition with artificial neural networks," Digital Signal Processing, vol. 20, no. 3, pp. 763-768, May 2010.

[8] R. Coggins, M. Jabri, B. Flower, and S. Pickard, "A hybrid analog and digital vlsi neural network for intracardiac morphology classification," IEEE Journal of Solid-state Circuits, vol. 30, no. 5, pp. 542-550, May 1995.

[9] A. Omondi and J. Rajapakse, FPGA Implementations of Neural Networks, Springer, 2006.

[10] Y. Maeda and M. Wakamura, "Simultaneous perturbation learning rule for recurrent neural networks and its fpga implementation," IEEE Trans. Neural Network, vol. 16, no. 6, pp. 1664$1672,2005$.

[11] S.P. Johnston, G. Prasad, L. Maguire, and T.M. Mcginnity, "An fpga hardware/software codesign towards evolvable spiking neural networks for robotics application," International Journal of Neural Systems, vol. 20, no. 6, pp. 447-461, December 2010.

[12] F.J. Lin, J.C. Hwang, P.H. Chou, and Y.C. Hung, "Fpga-based intelligent-complementary sliding-mode control for pmlsm servo-drive system," IEEE Trans. Power Electronics, vol. 25, no. 10, pp. 2573-2587, October 2010.

[13] A. Mellit, H. Mekki, A. Messai, and H. Salhi, "Fpga-based implementation of an intelligent simulator for stand-alone photovoltaic system," Expert Systems with Applications, vol. 37, no. 8, pp. 6036-6051, August 2010.

[14] F.M. Dias, A. Antunes, and A.M. Mota, "Artificial neural networks: a review of commercial hardware," Engineering Applications of Artificial Intelligence, vol. 17, no. 8, pp. 945-952, August 2004.

[15] J. Zhu and P. Sutton, "Fpga implementations of neural networks - a survey of a decade of progress," Proc. FPL'03, pp. 1062-1066, September 2003.

[16] L.M. Reyneri, "Implementation issues of neurofuzzy hardware: Going toward hw/sw codesign," IEEE Trans. Neural Network, vol. 14, no. 1, pp. 176-194, 2003.

[17] H. Li and S.D. Zhang, "A stochastic digital implementation of a neural network controller for small wind turbine systems," IEEE Trans. Power Electronics, vol. 21, no. 5, pp. 1502-1507, 2006.

[18] D. Ferrer and R. Gonzalez, "Neurofpga - implementing artificial neural networks on programmable logic devices," Proc. DATE'04, pp. 218-223, February 2004.

[19] S. Himavathi, "Feedforward neural network implementation in fpga using layer multiplexing for effective resource utilization," IEEE Trans. Neural Networks, vol. 18, no. 3, pp. 880-888, 2007.

[20] I.A. Basheer and M. Hajmeer, "Artificial neural networks: Fundamentals, computing, design, and application," Journal of Microbiological Methods, vol. 43, pp. 3-31, 2000.

[21] B. Muller, J. Reinhardt, and M. Strickland, Neural Networks: An Introduction (Physics of Neural Networks), Springer, 2002.

[22] T. Shima and M. Tukada, Neural Network and Neural device, Morikita publisher, 1997.

[23] Xilinx, http://www.xilinx.com/.

[24] A.K. Jain, R.P.W. Duin, and J.C. Mao, "Statistical pattern recognition: A review," IEEE Trans. Pattern Analysis and Machine Intelligence, vol. 22, no. 1, pp. 4-37, January 2000.

[25] A. Ouchar, R. Aksas, and H. Baudrand, "Artificial neural network for computing the resonant frequency of circular patch antennas," Microwave and optical technology letters, vol. 47, no. 6, pp. 564-566, October 2005.

[26] G.V. Puskorius and L.A. Feldkamp, "Neurocontrol of nonlinear dynamical systems with kalman filter trained recurrent networks," IEEE Trans. Neural Network, vol. 5, no. 2, pp. 279-297, March 1994.

[27] M.A. Kramer, "Nonlinear principal component analysis using autoassociative neural networks," Journal of AICHE, vol. 37, no. 2, pp. 233-243, 1991.

[28] M. Rajendra, P. Jena, and H. Raheman, "Prediction of optimized pretreatment process parameters for biodiesel production using ann and ga," Fuel, vol. 88, no. 5, pp. 868-875, 2009. 